\title{
LESÃO POR PRESSÃO EM IDOSOS NAS INSTITUIÇÕES DE LONGA PERMANÊNCIA: ATENUANTES NA PANDEMIA DA COVID-19
}

Juliana Balbinot Reis Girondi'

ORCID: 0000-0002-3763-4176

Karina Chamma Di Piero"

ORCID: 0000-0002-0102-6785

Cibely Freire de Oliveira"' ORCID: 0000-0003-3708-2719

Cristiane dos Santos Rosalv ORCID: 0000-0002-6001-7053

Luciana Alves dos Santos ${ }^{1 \mathrm{v}}$ ORCID: 0000-0002-7116-8930

Alcione Matos de Abreuv ORCID: 0000-0001-6078-7149

'Universidade Federal de Santa Catarina. Florianópolis, Santa Catarina, Brasil.

"Universidade Federal do Rio de Janeiro. Rio de Janeiro, Rio de Janeiro, Brasil.

"I'Residencial Israelita Albert Einstein. São Paulo, São Paulo, Brasil.

Iv Universidade Federal Fluminense. ILPI Espaço Vianney. Niterói, Rio de Janeiro, Brasil.

v Universidade Federal do Estado do Rio de Janeiro. Rio de Janeiro, Rio de Janeiro, Brasil.

Autor Correspondente: Juliana Balbinot Reis Girondi E-mail: juliana.balbinot@ufsc.br

\section{INTRODUÇÃO}

O envelhecimento da pele pode ser intrínseco e extrínseco; sendo a primeira causa relacionada a fatores genéticos e cronológicos, ao passo que o extrínseco resulta dos danos ambientais (radiação ultravioleta e outros fatores: tabagismo, etilismo, hábitos nutricionais dentre outros). Desse modo, as alterações cutâneas decorrentes do envelhecimento, quando associadas a fatores externos, como, por exemplo, pressão, umidade, trauma mecânico - fricção e cisalhamento podem potencializar as chances de ocorrerem Lesão por Pressão (LP) ${ }^{(1)}$.

Conceitualmente, LP é uma lesão localizada em uma área de dano da pele e do tecido mole subjacente geralmente sobre proeminência óssea ou relacionada com um dispositivo médico. A lesão pode apresentar pele intacta ou uma úlcera aberta. A lesão ocorre como resultado da intensa pressão e/ou prolongamento de pressão em combinação com cisalhamento. A tolerância do tecido mole para a pressão e cisalhamento também podem ser afetados pelo microclima, nutrição, perfusão, comorbidades e condições do tecido mole ${ }^{(2)}$

A pele é o maior órgão protetor do ser humano. No caso do idoso institucionalizado que vive em Instituição de Longa Permanência para Idosos ILPI, a LP é um problema de saúde, sobretudo idosos e para aqueles que passam maior parte do tempo acamados ou sentados, com exposição aos fatores de risco - extrínsecos e intrínsecos ${ }^{(3,4)}$.

Há vários desafios para implementar ações para reduzir a ocorrência da Lesão por Pressão nessa população, quais sejam: estruturais/processo de trabalho - reduzido número de profissionais da equipe de enfermagem, déficit/ dificuldade na educação permanente e baixa motivação desses para a implementação de protocolos de e/ou escalas preditivas de risco e para prevenção de Lesão por Pressão; e em relação à própria clientela atendida diferentes e elevados graus de dependência nos idosos, 
déficit cognitivo/aprendizagem, déficit de auto cuidado, diferentes graus de imobilidade, déficit de higiene pessoal e nutrição inadequada. ${ }^{(3,4)}$

Os idosos institucionalizados estão inseridos em um contexto de vulnerabilidades multidimensionais que perpassam por áreas sociais, culturais, políticas e econômicas, resultantes de fatores como aposentadoria, renda precária, discriminação, isolamento familiar e social, bem como o enfrentamento à fragilidade das políticas públicas para suporte dessa população vigente ${ }^{(5)}$.

Além dessas condições de vulnerabilidade aos quais os idosos institucionalizados estão submetidos, nos tempos atuais vêm requerendo maior vigilância e ações de cuidados a essa clientela; em virtude da COVID-19, que é uma doença infecciosa causada pelo vírus Severe Acute Respiratory Syndrome Coronavirus 2 (SARS-CoV-2() ${ }^{(6)}$. Entidades internacionais e nacionais elaboraram planos estratégicos para seu enfrentamento à saber:"COVID-19: Operational Planning Guidelines to Support Country Preparedness and Response"(7) criado pela OMS e "Plano de Contingência Nacional para Infecção Humana pelo novo Coronavírus COVID-19" elaborado pelo Ministério da Saúde.

A principal forma de contágio do novo coronavírus é pessoa-pessoa através de gotículas, aerossóis e contato com mãos, superfícies e objetos contaminados. Os principais sintomas são: febre, tosse seca, fadiga, perda de paladar e olfato, conjuntivite, congestão nasal, dores, diarreia, dor de garganta e erupção cutânea ${ }^{(8)}$.

Sobre as principais manifestações cutâneas da COVID-19, o enfermeiro deverá conhecer e durante o exame físico da pele dos idosos, realizar diagnóstico diferencial em relação às Lesões por Pressão. As principais lesões, determinadas pela manifestações cutâneas do novo coronavírus, são elas: erupções vesiculares, erupções com urticária, erupções maculo-papulares, pitiríase rósea, erupção perifolicular, eritema multiforme, eritema pseudovesicular, erupções purpúricas dissemidas e axilares, erupções morbilitiforme, eritema palmar, manchas livedoides e necrose ${ }^{(9)}$, essas são diferentes das Lesões por Pressão como podemos ver na Figura 1.
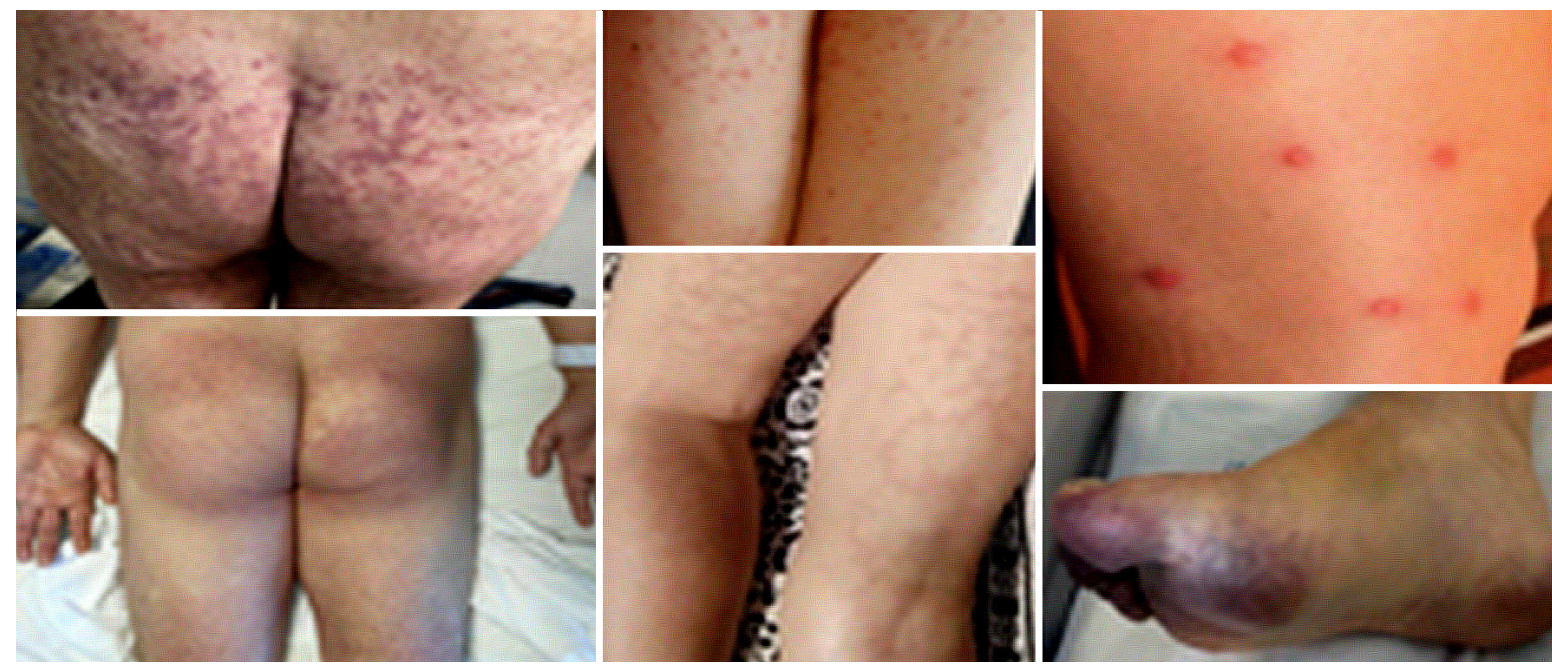

Figura 1 - Manifestações cutâneas da COVID 19. Arquivo Di Piero, 2020

Ainda sobre o quadro clínico da COVID-19, a maioria das pessoas com sintomas da doença não necessitam de hospitalização, entretanto pesquisas evidenciam que idosos acima de 60 anos com comorbidades tais como: hipertensão, diabetes mellitus, cardiopatia e doença renal; adultos jovens com imunossupressão; doentes crônicos; tabagistas e obesos são os mais susceptíveis ${ }^{(10)}$.

Diante disso, é relevante ressaltar a fragilidades dos idosos para risco de desenvolvimento da forma grave da doença e possíveis repercussões na pele decorrente da própria fisiopatologia da COVID-19, determinada pela hipóxia secundária à descompensação do aparelho respiratório, que entra em colapso com prejuízo da sua perfusão alvéolo-capilar e consequentemente, da perfusão tissular. Tal desencadear gera, pois, uma 
nutrição tecidual insuficiente, além da possibilidade da formação de micro trombos endovasculares, situação que também impede um fluxo circulatório satisfatório para a manutenção da nutrição da pele ${ }^{(11)}$.

Portanto, diante na situação pandêmica, deve-se reforçar o controle dos principais fatores de risco extrínsecos na prevenção do desenvolvimento de LP - pressão, fricção, alteração de microclima da pele (aumento de temperatura e umidade), atividade/mobilidade e hidratação/nutrição ${ }^{(2-4)}$.

Com isso, mediante cuidados de enfermagem sistematizados, o gerenciamento da pele frágil do idoso em ILPI deverá ser focado em três pilares - higienização, hidratação e análise/controle de risco conforme detalharemos nesse manuscrito.

\section{OBJETIVO}

Discutir sobre a prevenção da Lesão por Pressão em idosos institucionalizados em Instituições de Longa Permanência a partir do gerenciamento do cuidado de enfermagem durante a Pandemia da COVID-19.

\section{MÉTODO}

Estudo reflexivo que abordou referências teóricas sobre a prevenção de LP em idosos de ILPI considerando-se os atenuantes da pandemia COVID-19. Realizou-se uma revisão narrativa da literatura sobre as principais referências disponibilizadas em artigos científicos, manuais, protocolos institucionais, notas técnicas e documentos oficiais.

Posteriormente foi realizada uma discussão da importância da prevenção de LP em idosos institucionalizados, bem como a necessidade de estratégias para o gerenciamento do cuidado de enfermagem. A partir da análise o estudo possibilitou a construção de algumas etapas: diagnosticar uma situação problema para melhorar ou resolver; formular estratégias norteadoras para a implementação de ação; propor estratégias e sugerir meios de avaliação de sua eficiência possibilitando a ampliação e compreensão das necessidades de intervenção, considerando-se as peculiaridades de cada enfermeiro em seu contexto de atuação.

\section{GERENCIAMENTO DO CUIDADO DE ENFERMAGEM NA PREVENÇÃO DE LP EM IDOSOS INSTITUCIONALIZADOS DURANTE A PANDEMIA DA COVID-19}

Com vistas ao diagnóstico da situação problema, é fato que a Lesão por Pressão é um risco em pacientes idosos, conforme já amplamente difundido na literatura científica, tendo em vista a própria situação de declínio fisiológico e funcional, além das características de sua pele associado aos fatores extrínsecos e intrínsecos para risco de desenvolvimento de lesões. ${ }^{(2-4)}$

Dessa forma, o envelhecimento é um processo fisiológico, levando o corpo a diversas mudanças físicas e funcionais, e com o avançar da idade tendem a agravar, aumentando o risco para o surgimento de lesões por pressão. ${ }^{(2-4)}$

Com a pandemia do novo coronavírus tal situação é agravada, tendo em vista as situações relacionadas à própria doença - hipóxia e micro trombos, os quais podem gerar redução de perfusão tecidual e aumento de áreas de risco, que se somadas ao imobilismo, a pressão em áreas de superfícies rígidas sem o equilíbrio de pressão adequado e técnicas de descompressão sistematizadas por equipe treinada, pode gerar lesões que aumentam a morbimortalidade desse idoso.

Tendo em vista, que a pele é considerada o maior órgão do corpo humano e têm funções vitais como revestimento e a sustentação de estruturas internas; proteção contra raios ultravioleta, traumas físicos, químicos, inclusive contra a ação de microrganismos patogênicos; responsável pela termorregulação, percepção sensorial, equilíbrio hídrico, imagem corporal e metabolismo como a síntese de vitamina $\mathrm{D}^{(12)}$, sua avaliação diária através do exame clínico pelo enfermeiro é fundamental para avaliação de alterações de sua integridade. 
O cuidado e a proteção da pele dos idosos em ILPI é um desafio constante para o enfermeiro, visto que a pele desta clientela é frágil e possuem os fatores de risco inerentes, como extremo de idade, estado nutricional deficiente, doenças crônicas, polifarmácia, declínio cognitivo, sensorial visual e mobilidade diminuída.

Compete ao enfermeiro e sua equipe, a inspeção e descrição individualizada dos fatores de risco para desenvolver Lesão por Pressão, registrando os aspectos fundamentais para a prevenção, monitorar e documentar as intervenções/ações, bem como avaliar os resultados obtidos com o cuidado planejado.

Diante disso, devem ser organizadas com base na Sistematização da Assistência em Enfermagem (SAE) estratégias de cuidados preventivos, que a partir de evidências científicas irão fundamentar o processo de enfermagem e a prática de enfermagem em ILPI.

Dessa forma apresenta-se no Quadro 1 os pilares para prevenção da Lesão por Pressão, assim como as principais medidas interventivas protetoras; possibilidades de execução e reavaliação.

Quadro 1 - Estratégias de ação para gerenciamento do cuidado de enfermagem na prevenção de LP em idosos institucionalizados durante a Pandemia da Covid-19

\begin{tabular}{|c|c|c|c|c|c|}
\hline $\begin{array}{l}\text { Pilares da } \\
\text { prevenção }\end{array}$ & $\begin{array}{l}\text { Medidas } \\
\text { preventivas }\end{array}$ & Método & Responsável & $\begin{array}{l}\text { Período de } \\
\text { realização }\end{array}$ & Reavaliação \\
\hline HIGIENE & $\begin{array}{l}\text { Higienizar a } \\
\text { pele }\end{array}$ & $\begin{array}{l}\text { - Higienizar a pele com produtos sem } \\
\text { álcool e sem perfume, levemente } \\
\text { ácido (pH em torno de 5,5). Preferir } \\
\text { sabonete líquido ou limpador de pele } \\
\text { industrializado sem enxague com } \\
\text { dimeticona. } \\
\text { - Utilizar toalhas de banho com fibras } \\
\text { macias. } \\
\text { - Atentar para a temperatura da } \\
\text { água, evitando altas temperaturas, } \\
\text { minimizando os riscos de ressecamento } \\
\text { da pele e predisposição a lesões. } \\
\text { - Quando utilização de lenços } \\
\text { umedecidos/banho seco, optar por } \\
\text { produtos à base de dimeticona, aloe } \\
\text { vera e sem álcool. } \\
\text { - Realizar manejo gentil durante a } \\
\text { higiene da pele da área íntima - genito- } \\
\text { urinária sempre que estiver em contato } \\
\text { com excretas, em especial a região } \\
\text { perianal, evitando lesões. }\end{array}$ & $\begin{array}{l}\text { Enfermeiro, } \\
\text { equipe de } \\
\text { enfermagem } \\
\text { e cuidadores. }\end{array}$ & $\begin{array}{l}\text { Diariamente e } \\
\text { a cada troca de } \\
\text { fralda. }\end{array}$ & $\begin{array}{l}\text { Diária/Sempre } \\
\text { que houver } \\
\text { necessidade. }\end{array}$ \\
\hline \multirow[b]{2}{*}{ HIDRATAÇÃO } & Hidratar a pele & $\begin{array}{l}\text { - Utilizar cremes hidratantes ou loções } \\
\text { hidratantes que hidratem e umectem a } \\
\text { pele. Atenção! Não massagear áreas de } \\
\text { proeminências ósseas e hiperemiadas, } \\
\text { pois aumentam o risco de lesão. }\end{array}$ & $\begin{array}{l}\text { Enfermeiro, } \\
\text { equipe de } \\
\text { enfermagem } \\
\text { e cuidadores. }\end{array}$ & $\begin{array}{l}\text { Duas vezes ao dia } \\
\text { (preferencialmente } \\
\text { após higiene). }\end{array}$ & $\begin{array}{l}\text { Diariamente } \\
\text { conforme } \\
\text { prescrição do } \\
\text { enfermeiro. }\end{array}$ \\
\hline & $\begin{array}{l}\text { Otimizar } \\
\text { hidratação } \\
\text { oral e } \\
\text { suplementação } \\
\text { nutricional. }\end{array}$ & $\begin{array}{l}\text { - Estimular a ingesta hídrica e aceitação } \\
\text { alimentar adequada às necessidades } \\
\text { nutricionais do idoso. } \\
\text { - Avaliar conforme exames laboratoriais } \\
\text { junto à equipe médica e de nutrição, a } \\
\text { introdução de suplementação calórico/ } \\
\text { proteica/oligoelementos. }\end{array}$ & $\begin{array}{l}\text { Enfermeiro, } \\
\text { Médico e } \\
\text { Nutricionista. }\end{array}$ & Diariamente. & Semanalmente. \\
\hline $\begin{array}{l}\text { ANÁLISE E } \\
\text { CONTROLE } \\
\text { DE RISCOS }\end{array}$ & $\begin{array}{l}\text { Inspecionar a } \\
\text { pele }\end{array}$ & $\begin{array}{l}\text { - Realizar exame físico da pele, para avaliar } \\
\text { e registrar presença de alterações na } \\
\text { estrutura da pele, coloração, umidade, etc. } \\
\text { Atenção! Preferir o momento da higiene } \\
\text { para otimizar a avaliação. }\end{array}$ & $\begin{array}{l}\text { Enfermeiro, } \\
\text { equipe de } \\
\text { enfermagem } \\
\text { e cuidadores. }\end{array}$ & Nas 24 horas. & $\begin{array}{l}\text { A cada } \\
\text { demanda } \\
\text { apresentada. }\end{array}$ \\
\hline
\end{tabular}


Continuação do Quadro 1

\begin{tabular}{|c|c|c|c|c|c|}
\hline $\begin{array}{l}\text { Pilares da } \\
\text { prevenção }\end{array}$ & $\begin{array}{l}\text { Medidas } \\
\text { preventivas }\end{array}$ & Método & Responsável & $\begin{array}{l}\text { Período de } \\
\text { realização }\end{array}$ & Reavaliação \\
\hline \multirow{2}{*}{$\begin{array}{l}\text { ANÁLISE E } \\
\text { CONTROLE } \\
\text { DE RISCOS }\end{array}$} & $\begin{array}{l}\text { Realizar } \\
\text { busca ativa } \\
\text { de fatores de } \\
\text { riscos }\end{array}$ & $\begin{array}{l}\text { - Exame físico: inspecionar a pele. } \\
\text { - Identificar fatores de riscos e prescrever } \\
\text { conforme diagnósticos de enfermagem } \\
\text { levantados, as intervenções para o } \\
\text { enfrentamento destes. }\end{array}$ & Enfermeiro. & $\begin{array}{l}\text { No acolhimento/ } \\
\text { admissão do } \\
\text { idoso na ILPI e } \\
\text { em avaliações } \\
\text { periódicas. }\end{array}$ & $\begin{array}{l}\text { Diariamente e a } \\
\text { cada mudança } \\
\text { clínica do idoso. }\end{array}$ \\
\hline & $\begin{array}{l}\text { Identificar o } \\
\text { grau de risco } \\
\text { preditivo de } \\
\text { LP }\end{array}$ & - Aplicar a escala de Braden (Foto 4) & Enfermeiro. & $\begin{array}{l}\text { No acolhimento } \\
\text { do idoso } \\
\text { (preferencialmente } \\
\text { nas } 6 \text { primeiras } \\
\text { horas). }\end{array}$ & $\begin{array}{l}\text { Após a } \\
\text { admissão, a } \\
\text { cada mudança } \\
\text { clínica, ou } \\
\text { pelo menos } \\
\text { semanalmente }\end{array}$ \\
\hline \multirow[t]{2}{*}{$\begin{array}{l}\text { ANÁLISE E } \\
\text { CONTROLE } \\
\text { DE RISCOS }\end{array}$} & $\begin{array}{l}\text { Manejar } \\
\text { umidade da } \\
\text { pele. }\end{array}$ & $\begin{array}{l}\text { - Utilizar produtos à base de óxido de } \\
\text { zinco e petrolatum, dimeticona, alloe vera, } \\
\text { soluções poliméricas, entre outras; para } \\
\text { determinar uma barreira protetora na pele } \\
\text { contra excesso de umidade. } \\
\text { - Remover fralda úmida e com sujidades } \\
\text { sempre que houver saturação do SAP } \\
\text { (gel super absorvente) demonstrado no } \\
\text { indicador de umidade (traço mediano que } \\
\text { altera de cor conforme necessidade da } \\
\text { troca da fralda). } \\
\text { Atenção! Não utilizar mais de uma fralda e } \\
\text { não associar uso de absorvente com fralda } \\
\text { e nem absorvente com calça absorvente. } \\
\text { Avaliar o grau de incontinência e identificar } \\
\text { a necessidade do melhor dispositivo } \\
\text { absorvente individualmente. } \\
\text { - Avaliar uso de comadres, patinhos, } \\
\text { dispositivos externos de incontinência } \\
\text { urinária masculina e cateter vesical de } \\
\text { demora/dispositivos anal em incontinentes. } \\
\text { - Realizar troca de roupas de cama e traçado } \\
\text { móvel sempre que molhados ou com } \\
\text { sujidades. } \\
\text { - Atentar para outras fontes de umidade: } \\
\text { drenos sobre a pele, exsudato de feridas, } \\
\text { febre, suor e transudato em pacientes com } \\
\text { anasarca/linfedema. }\end{array}$ & $\begin{array}{l}\text { Enfermeiro, } \\
\text { equipe de } \\
\text { enfermagem } \\
\text { cuidadores. }\end{array}$ & $\begin{array}{l}\text { Uma vez ao dia } \\
\text { após o banho e } \\
\text { após trocas de } \\
\text { fraldas (realizada } \\
\text { conforme grau } \\
\text { de incontinência } \\
\text { e necessidade da } \\
\text { troca de fralda/ } \\
\text { higiene íntima. }\end{array}$ & $\begin{array}{l}\text { Após troca de } \\
\text { fralda e higiene } \\
\text { íntima. } \\
\text { Periodicamente } \\
\text { de acordo } \\
\text { com saturação } \\
\text { da fralda } \\
\text { (determinada } \\
\text { pelo indicador } \\
\text { de umidade da } \\
\text { fralda). }\end{array}$ \\
\hline & $\begin{array}{l}\text { Reduzir } \\
\text { fricção e } \\
\text { cisalhamento }\end{array}$ & $\begin{array}{l}\text { - Realizar elevação da cabeceira da cama } \\
\text { até no máximo } 30^{\circ} \text {. } \\
\text { - Realizar lateralização quando nos } \\
\text { decúbitos laterais direito e esquerdo, } \\
\text { angulando em } 30 \text { graus com utilização de } \\
\text { travesseiro/rolinho sob a área do dorso. } \\
\text { Atenção! Não posicionar o paciente sobre a } \\
\text { área do trocânter. } \\
\text { - Limitar o tempo de cabeceira elevada (90 } \\
\text { graus), preferencialmente apenas } 1 \text { hora, } \\
\text { para evita fricção } \\
\text { e cisalhamento. } \\
\text { - Aplicar traçado móvel ou dispositivo } \\
\text { mecânico de elevação para mover } \\
\text { pacientes acamados durante transferências } \\
\text { e alternâncias de decúbito. }\end{array}$ & $\begin{array}{l}\text { Enfermeiro, } \\
\text { equipe de } \\
\text { Enfermagem } \\
\text { e cuidadores }\end{array}$ & Nas 24 horas & Nas 24 horas \\
\hline
\end{tabular}


Continuação do Quadro 1

\begin{tabular}{|c|c|c|c|c|c|}
\hline $\begin{array}{l}\text { Pilares da } \\
\text { prevenção }\end{array}$ & $\begin{array}{l}\text { Medidas } \\
\text { preventivas }\end{array}$ & Método & Responsável & $\begin{array}{l}\text { Período de } \\
\text { realização }\end{array}$ & Reavaliação \\
\hline $\begin{array}{l}\text { ANÁLISE E } \\
\text { CONTROLE } \\
\text { DE RISCOS }\end{array}$ & $\begin{array}{l}\text { Reduzir } \\
\text { pressão }\end{array}$ & $\begin{array}{l}\text { - Utilizar colchões de pressão alternada ou } \\
\text { viscoelástico. } \\
\text { Atenção! Não utilizar colchões piramidais, } \\
\text { de água, gel e de espuma padrão, pois não } \\
\text { redistribuem a pressão corporal. } \\
\text { - Utilizar coxins, reposicionadores, rolinhos } \\
\text { e travesseiros para reposicionar a cada } 2 \\
\text { horas, angulando em } 30 \text { graus o corpo } \\
\text { do idoso - Evitar posicionar o idoso sobre } \\
\text { proeminências ósseas. } \\
\text { - Elevar calcâneos com rolinho ou } \\
\text { travesseiro sob panturrilhas. }\end{array}$ & $\begin{array}{l}\text { Enfermeiro, } \\
\text { equipe de } \\
\text { Enfermagem } \\
\text { e cuidadores }\end{array}$ & $\begin{array}{l}\text { Nas } 24 \text { horas } \\
\text { (Atenção! A } \\
\text { mudanla de } \\
\text { decúbito deve ser } \\
\text { realizada } 2 / 2 ; \text { na } \\
\text { cadeira o idoso só } \\
\text { pode permanecer } \\
\text { até } 1 \text { hora } \\
\text { sentado) }\end{array}$ & $\begin{array}{l}\text { Conforme } \\
\text { prescrição do } \\
\text { enfermeiro }\end{array}$ \\
\hline
\end{tabular}

Medidas preventivas como a aplicação de escalas, abrangem ações simples e demandam poucos gastos, e devem ser incentivadas no âmbito das ILPI para a prevenção de LP. A Escala de Braden tem sido muito utilizada em pesquisas nacionais com idosos institucionalizados e apresenta elevada sensibilidade e especificidade na predição de risco para o desenvolvimento de LP.(4)É uma escala também indicada pela NPIAP/ EPUAP para prevenção de LP, tanto para adultos quanto para idosos. ${ }^{(10)}$

Os cuidados essenciais para a manutenção da integridade cutânea que inclui a inspeção da pele em intervalos pré-definidos, cuja periodicidade é proporcional ao risco identificado de acordo com os resultados encontrados na escala de Braden, sendo necessário o registro apropriado e pontual das alterações encontradas.

A COVID-19 em suas manifestações distintas exige do profissional conhecimento prévio, para diagnóstico diferencial das lesões que podem surgir na pele do idoso com a infecção do novo coronavírus, além de um olhar atento aos achados relacionados aos fatores de risco para LP.

A abordagem sistematizada para o tratamento de feridas conforme a ferramenta TIME ${ }^{(12)}$, define conforme as fases de cicatrização de uma ferida, condutas/orientações que podem auxiliar o enfermeiro a garantir o avanço na cicatrização das feridas, por ele acompanhadas.

Primeiramente, a ferida deve ser analisada com base no seu diagnóstico etiológico, o que gera embasamento clínico para entender sua causa, e diante disso, compreender a evolução clínica, que analisada macroscopicamente, normalmente inicia-se com tecido desvitalizado (não viável) decorrente de ação nociva, no caso da LP, decorrente de pressão, fricção, cisalhamento e/ou alteração do microclima.

Conforme a ferramenta ${ }^{(11)}$ o tecido inviável (desvitalizado - necrosado, normalmente de cor preta, marrom ou amarela) deve ser debridrado instrumentalmente pelo enfermeiro capacitado em debridamento, ou manejado com debridantes químicos tópicos. Diante da lesão debridada, já sem o tecido inviável, se inflamado (com sinais de inflamação)e/ou infecção (presença de sinais clínicos de infecção de ferida superficial/profunda/ sistêmica), a ação portanto deverá ser o controle dessa complicação mediante coberturas específicas para processos inflamatórios agudo ou crônicos de feridas, realização de técnicas para melhora desta situação ou ainda intervenção médica para introdução de medicações sistêmica.

Uma vez controlada a inflamação/infecção, evolui-se com a necessidade de controle adequado da umidade do leito da ferida, também com auxílio de tecnologias mediante utilização de coberturas de alta complexidade e por fim, já na fase final de remodelamento da ferida, o controle final será das margens ou bordas da ferida para finalizar seu fechamento, por contração eficiente, com a conclusão do reparo ou cicatrização da ferida.

\section{Limitações}

No caso de falha das medidas de prevenção, recomenda-se a avaliação de um enfermeiro especialista em enfermagem dermatológica/ estomaterapeuta, a fim de realizar a avaliação baseada no processo de enfermagem que será voltada tanto para o paciente quanto para lesão. 


\section{Implicações para a prática}

A partir das estratégias propostas sugere-se o desenvolvimento e acompanhamento das ações pelo enfermeiro, que apesar de serem ações simples, são efetivas na prevenção dessas lesões. Diante disso, os enfermeiros atuantes em ILPI, precisam liderar com base na educação o consenso de saberes e práticas para prevenção de LP considerando os atenuantes da COVID-19 em idosos institucionalizados.

\section{CONSIDERAÇÕES FINAIS}

A partir da proposta apresentada foi possível nortear uma reflexão sobre a prevenção de Lesão por Pressão em idosos institucionalizados, considerando a importância do gerenciamento do cuidado de enfermagem frente aos atenuantes da Pandemia da Covid-19.

Foram propostas pelo estudo, estratégias com base na avaliação clínica, aplicação da escala de Braden, diagnóstico de enfermagem e ações diárias por meio da prescrição de enfermagem, direcionadas para o controle dos principais fatores de risco apresentados individualmente nos idosos institucionalizados com vistas ao controle do surgimento da Lesão por Pressão.

Além disso, foi referida a importância da implementação conjugada à diária do cumprimento das ações pela equipe de enfermagem, com inclusive, melhor compreensão das situações apresentadas e necessidades de ajustes institucionais com base em estratégias educacionais que deverão subsidiar mudanças de comportamento e cultura de manejo desse problema de saúde pública, e que também aponta para questões relacionadas a qualidade da assistência prestada pela equipe de saúde na ILPI.

\section{AGRADECIMENTO}

Ao Departamento Científico de Enfermagem Gerontológica da ABEn Nacional.

\section{REFERÊNCIAS}

1. Santos $C T$, Almeida MA, Lucena AF. Diagnóstico de enfermagem risco de úlcera por pressão: validação de conteúdo. Rev Latino-Am Enfermagem [Internet]. 2016 [cited 2020 Mai 12];24: e2693. Available from: https://www.scielo.br/pdf/rlae/v24/ pt_0104-1169-rlae-24-02693.pdf. DOI: http://dx.doi.org/10.1590/1518-8345.0782.2693

2. National Pressure Ulcer Advirosy Panel. Pressure Injury Stages [Internet]. 2016. [cited 2020 Abr 11]. Available from: http:// www.npuap.org/resources/educational-and-clinical-resources/npuap-pressure-injury-stages/

3. Vieira VAS, Santos MDC, Almeida NA, et al. Risco de lesão por pressão em idosos com comprometimento na realização de atividades diárias. Revista de Enfermagem do Centro-Oeste Mineiro[Internet]. 2018. [cited 2020 Jun 26]; 8:e2599. Available from: http://seer.ufsj.edu.br/index.php/recom/article/view/2599. DOI: http://dx.doi.org/10.19175/recom.v7i0.2599

4. Macêdo PKG, Anízio BKF, Brito FM et al. Instrumento de coleta de dados para prevenção de úlcera por pressão no idoso institucionalizado. Rev enferm UFPE on line., Recife, 10(11):3977-88, nov., 2016. [cited 2020 Jul 28]. DOI: 10.5205/ reuol.9881-87554-1-EDSM1011201620

5. Cruz RR, Beltrame V, Dallacosta FM. Envelhecimento e vulnerabilidade: análise de 1.062 idosos. Rev bras geriatr gerontol [Internet]. 2019 [cited 2020 June 30];22(3):e180212.Available from: http://www.scielo.br/ scielo.php?script=sci_arttext\&pid=S1809-98232019000300204\&Ing=en. Epub Sep 26, 2019. DOI: https://doi. org/10.1590/1981-22562019022.180212.

6. Albuquerque NLS de. Planejamento operacional durante a pandemia de Covid-19: comparação entre recomendações da Organização Mundial da Saúde e o Plano de Contingência Nacional. Cogitare enferm. [Internet]. 2020 [acesso em 03 de mai 2020]; 25. Disponível em: http://dx.doi.org/10.5380/ce.v25i0.72659

7. World Health Organization. COVID-19 Strategic preparedness and response plan operational planning guidelines to support country preparedness and response. Geneva: WHO; 2020. 
8. Organização Pan Americana de Saúde (OPAS). Folha informativa - covid-19 (doença causada pelo novo coronavírus). [Internet] [acesso 01 de mai 2020]. Disponível em: https://www.paho.org/bra/index.php?option=com_content\&view=artic le\&id=6101: covid19\&ltemid=875

9. Casas, C. et al; .Classification of the cutaneous manifestations of COVID-19: a rapid prospective nationwide consensus study in spain with 375 cases. Supplementary material: Photographic Atlas. British Journal of Dermatology 2020. [Internet]. Available from: https://onlinelibrary.wiley.com/doi/epdf/10.1111/bjd.19163

10. National Pressure Ulcer Advirosy Panel. Pressure Injury Stages. Unavoidable Pressure Injury during COVID-19 Pandemic: A Position Paper from the National Pressure Injury Advisory Panel [Internet]. 2020. [cited 2020 Abr 11]. Available from: https://cdn.ymaws.com/npiap.com/resource/resmgr/white_papers/Unavoidable_in_COVID_Pandemi.pdf

11. Mittag BF, Krause TCC, Roehrs H, Meier MJ, Danski MTR. Cuidados com Lesão de Pele: Ações da Enfermagem. Estima [Internet]. 2017. [cited 2020 Mai 26]; 15(1): 19-25. Available from: https://pdfs.semanticscholar.org/f8b5/ be99de89bd410cff34d93eb9e982bdfa79bf.pdf. DOI: 10.5327/Z1806-3144201700010004

12. Utilizar a abordagem sistematizada no tratamento de feridas: Wound Bed Preparation (WBP) e a ferramenta TIME.Schultz GS, Barillo DJ, Mozingo DW, Chin GA. Wound bed preparation and a brief history of TIME. International Wound Journal. 2004;1:19-31. 